Texts in Applied Mathematics 32

Editors

J.E. Marsden

L. Sirovich

M. Golubitsky

W. Jäger

Advisors

G. Iooss

P. Holmes

D. Barkley

M. Dellnitz 


\section{Texts in Applied Mathematics}

1. Sirovich: Introduction to Applied Mathematics.

2. Wiggins: Introduction to Applied Nonlinear Dynamical Systems and Chaos, 2nd ed.

3. Hale/Koçak: Dynamics and Bifurcations.

4. Chorin/Marsden: A Mathematical Introduction to Fluid Mechanics, 3rd ed.

5. Hubbard/West: Differential Equations: A Dynamical Systems Approach: Ordinary Differential Equations.

6. Sontag: Mathematical Control Theory: Deterministic Finite Dimensional Systems, 2nd ed.

7. Perko: Differential Equations and Dynamical Systems, 3rd ed.

8. Seaborn: Hypergeometric Functions and Their Applications.

9. Pipkin: A Course on Integral Equations.

10. Hoppensteadt/Peskin: Modeling and Simulation in Medicine and the Life Sciences, 2nd ed.

11. Braun: Differential Equations and Their Applications, 4th ed.

12. Stoer/Bulirsch: Introduction to Numerical Analysis, 3rd ed.

13. Renardy/Rogers: An Introduction to Partial Differential Equations, 2nd ed.

14. Banks: Growth and Diffusion Phenomena: Mathematical Frameworks and Applications.

15. Brenner/Scott: The Mathematical Theory of Finite Element Methods, 2nd ed.

16. Van de Velde: Concurrent Scientific Computing.

17. Marsden/Ratiu: Introduction to Mechanics and Symmetry, 2nd ed.

18. Hubbard/West: Differential Equations: A Dynamical Systems Approach: HigherDimensional Systems.

19. Kaplan/Glass: Understanding Nonlinear Dynamics.

20. Holmes: Introduction to Perturbation Methods.

21. Curtain/Zwart: An Introduction to Infinite-Dimensional Linear Systems Theory.

22. Thomas: Numerical Partial Differential Equations: Finite Difference Methods.

23. Taylor: Partial Differential Equations: Basic Theory.

24. Merkin: Introduction to the Theory of Stability of Motion.

25. Naber: Topology, Geometry, and Gauge Fields: Foundations.

26. Polderman/Willems: Introduction to Mathematical Systems Theory: A Behavioral Approach.

27. Reddy: Introductory Functional Analysis with Applications to Boundary-Value Problems and Finite Elements.

28. Gustafson/Wilcox: Analytical and Computational Methods of Advanced Engineering Mathematics.

29. Tveito/Winther: Introduction to Partial Differential Equations: A Computational Approach.

30. Gasquet/Witomski: Fourier Analysis and Applications: Filtering, Numerical Computation, Wavelets. 


\author{
Dale R. Durran
}

\title{
Numerical Methods for Wave Equations in Geophysical Fluid Dynamics
}

With 93 Illustrations 
Dale R. Durran

Atmospheric Sciences

University of Washington

Box 351640

Seattle, WA 98195-1640

USA

\section{Series Editors}

J.E. Marsden

Control and Dynamical Systems, 107-81

California Institute of Technology

Pasadena, CA 91125

USA

M. Golubitsky

Department of Mathematics

University of Houston

Houston, TX 77204-3476

USA

\section{Sirovich}

Division of Applied Mathematics

Brown University

Providence, RI 02912

USA

\author{
W. Jäger \\ Department of Applied Mathematics \\ Universität Heidelberg \\ Im Neuenheimer Feld 294 \\ 69120 Heidelberg \\ Germany
}

Mathematics Subject Classification (1991): 65Mxx, 86A10, 76-01, 35L05

Library of Congress Cataloging-in-Publication Data

Durran, Dale R.

Numerical methods for wave equations in geophysical fluid dynamics

/ Dale R. Durran.

p. cm.-(Texts in applied mathematics ; 32)

Includes bibliographical references and index.

ISBN 978-1-4419-3121-4 ISBN 978-1-4757-3081-4 (eBook)

DOI 10.1007/978-1-4757-3081-4

1. Fluid dynamics-Methodology. 2. Geophysics-Methodology.

3. Wave equation. 4. Numerical analysis. 5. Differential equations, Partial-Numerical solutions. I. Title. II. Series

QC809.F5D87 1998

$550^{\prime} .1^{\prime} 532059-\mathrm{dc} 21$

98-24739

Printed on acid-free paper.

(C) 1999 Springer Science+Business Media New York

Originally published by Springer-Verlag New York, Inc in 1999.

Softcover reprint of the hardcover 1st edition 1999

All rights reserved. This work may not be translated or copied in whole or in part without the written permission of the publisher (Springer-Verlag New York, Inc., 175 Fifth Avenue, New York, NY 10010, USA), except for brief excerpts in connection with reviews or scholarly analysis. Use in connection with any form of information storage and retrieval, electronic adaptation, computer software, or by similar or dissimilar methodology now known or hereafter developed is forbidden.

The use of general descriptive names, trade names, trademarks, etc., in this publication, even if the former are not especially identified, is not to be taken as a sign that such names, as understood by the Trade Marks and Merchandise Marks Act, may accordingly be used freely by anyone.

987655432

Springer-Verlag is a part of Springer Science + Business Media

springeronline.com 
To every hand that's touched the Wall 


\section{Series Preface}

Mathematics is playing an ever more important role in the physical and biological sciences, provoking a blurring of boundaries between scientific disciplines and a resurgence of interest in the modern as well as the classical techniques of applied mathematics. This renewal of interest, both in research and teaching, has led to the establishment of the series: Texts in Applied Mathematics (TAM).

The development of new courses is a natural consequence of a high level of excitement on the research frontier as newer techniques, such as numerical and symbolic computer systems, dynamical systems, and chaos, mix with and reinforce the traditional methods of applied mathematics. Thus, the purpose of this textbook series is to meet the current and future needs of these advances and encourage the teaching of new courses.

$T A M$ will publish textbooks suitable for use in advanced undergraduate and beginning graduate courses, and will complement the Applied Mathematical Sciences (AMS) series, which will focus on advanced textbooks and research level monographs. 


\section{Preface}

This book is designed to serve as a textbook for graduate students or advanced undergraduates studying numerical methods for the solution of partial differential equations governing wave-like flows. Although the majority of the schemes presented in this text were introduced in either the applied-mathematics or atmospheric-science literature, the focus is not on the nuts-and-bolts details of various atmospheric models but on fundamental numerical methods that have applications in a wide range of scientific and engineering disciplines. The prototype problems considered include tracer transport, shallow-water flow and the evolution of internal waves in a continuously stratified fluid.

A significant fraction of the literature on numerical methods for these problems falls into one of two categories, those books and papers that emphasize theorems and proofs, and those that emphasize numerical experimentation. Given the uncertainty associated with the messy compromises actually required to construct numerical approximations to real-world fluid-dynamics problems, it is difficult to emphasize theorems and proofs without limiting the analysis to classical numerical schemes whose practical application may be rather limited. On the other hand, if one relies primarily on numerical experimentation it is much harder to arrive at conclusions that extend beyond a specific set of test cases. In an attempt to establish a clear link between theory and practice, I have tried to follow a middle course between the theorem-and-proof formalism and the reliance on numerical experimentation. There are no formal proofs in this book, but the mathematical properties of each method are derived in a style familiar to physical scientists. At the same time, numerical examples are included that illustrate these theoretically derived properties and facilitate the intercomparison of various methods. 
A general course on numerical methods for geophysical fluid dynamics might draw on portions of the material presented in Chapters 2 through 6. Chapter 2 describes the largely classical theory of finite-difference approximations to the oneway wave equation (or alternatively the constant-wind-speed advection equation). The extension of these results to systems of equations, several space dimensions, dissipative flows and nonlinear problems is discussed in Chapter 3. Chapter 4 introduces series-expansion methods with emphasis on the Fourier and sphericalharmonic spectral methods and the finite-element method. Finite-volume methods are discussed in Chapter 5 with particular attention devoted to methods for simulating the transport of scalar fields containing poorly resolved spatial gradients. Semi-Lagrangian schemes are analyzed in Chapter 6. Both theoretical and applied problems are provided at the end of each chapter. Those problems that require numerical computation are marked by an asterisk.

In addition to the core material in Chapters 2 through 6, the introduction in Chapter 1 discusses the relation between the equations governing wave-like geophysical flows and other types of partial differential equations. Chapter 1 concludes with a short overview of the strategies for numerical approximation that are considered in detail throughout the remainder of the book. Chapter 7 examines schemes for the approximation of slow moving waves in fluids that support physically insignificant fast waves. The emphasis in Chapter 7 is on atmospheric applications in which the slow wave is either an internal gravity wave and the fast waves are sound waves, or the slow wave is a Rossby wave and the fast waves are both gravity waves and sound waves. Chapter 8 examines the formulation of wave-permeable boundary conditions for limited-area models with emphasis on the shallow-water equations in one and two dimensions and on internally stratified flow.

Many numerical methods for the simulation of internally stratified flow require the repeated solution of elliptic equations for pressure or some closely related variable. Due to the limitations of my own expertise and to the availability of other excellent references I have not discussed the solution of elliptic partial differential equations in any detail. A thumbnail sketch of some solution strategies is provided in Section 7.1.3; the reader is referred to Chapter 5 of Ferziger and Perić (1997) for an excellent overview of methods for the solution of elliptic equations arising in computational fluid dynamics.

I have attempted to provide sufficient references to allow the reader to further explore the theory and applications of many of the methods discussed in the text, but the reference list is far from encyclopedic and certainly does not include every worthy paper in the atmospheric science or applied mathematics literature. References to the relevant literature in other disciplines and in foreign language journals is rather less complete. ${ }^{1}$

\footnotetext{
${ }^{1}$ Those not familiar with the atmospheric science literature may be surprised by the number of references to Monthly Weather Review, which despite its title, has become the primary American journal for the publication of papers on numerical methods in atmospheric science.
} 
This book would not have been written without the generous assistance of several colleagues. Christopher Bretherton, in particular, provided many perceptive answers to my endless questions. J. Ray Bates, Byron Boville, Michael Cullen, Marcus Grote, Robert Higdon, Randall LeVeque, Christoph Schär, William Skamarock, Piotr Smolarkiewicz, and David Williamson all provided very useful comments on individual chapters. Many students used earlier versions of this manuscript in my courses in the Atmospheric Sciences Department at the University of Washington, and their feedback helped improve the clarity of the manuscript. Two students to whom I am particularly indebted are Craig Epifanio and Donald Slinn. I am also grateful to James Holton for encouraging me to undertake this project.

It is my pleasure to acknowledge the many years of support for my numerical modeling efforts provided by the Mesoscale Dynamic Meteorology Program of the National Science Foundation. Additional support for my atmospheric simulation studies has been provided by the Coastal Meteorology ARI of the Office of Naval Research. Part of this book was completed while I was on sabbatical at the Laboratoire d'Aérologie of the Université Paul Sabatier in Toulouse, France, and I thank Daniel Guedalia and Evelyne Richard for helping make that year productive and scientifically stimulating.

As errors in the text are identified, they will be posted on the web at http:// www. atmos.washington.edu/methods.for.waves, which can be accessed directly or via Springer's home page at http://www.springer-ny.com. I would be most grateful to be advised of any typographical or other errors by electronic mail at dale.durran@atmos.washington.edu.

Seattle, Washington

DALE R. DURRAN

Cover art: The three curves plot solutions to the linearized Rossby-adjustment problem. The governing equations and physical parameters for this problem are identical to those given in Problem 12 of Chapter 3, except that the spatial domain is $-400 \mathrm{~km} \leq x \leq 400 \mathrm{~km}$ with open lateral boundaries, and the initial condition for the free-surface displacement is $h(x, t=0)=\arctan (x / 20 \mathrm{~km})$. The curves shown are plots of $u(x, t=943 \mathrm{~s}), u(x, t=1222 \mathrm{~s})$, and $u(x, t=1501 \mathrm{~s})$ on an artisically cropped portion of the sub-domain $x>0$. 


\section{Contents}

Series Preface vii

$\begin{array}{ll}\text { Preface } & \text { ix }\end{array}$

1 Introduction 1

1.1 Partial Differential Equations-Some Basics . . . . . . . . . 2

1.1.1 First-Order Hyperbolic Equations . . . . . . . . . . 4

1.1.2 Linear Second-Order Equations in Two Independent Variables . . . . . . . . . . . 7

1.2 Wave Equations in Geophysical Fluid Dynamics . . . . . . . . 11

1.2.1 Hyperbolic Equations . . . . . . . . . . . . 12

1.2 .2 Filtered Equations . . . . . . . . . . . . 20

1.3 Strategies for Numerical Approximation . . . . . . . . . . . 26

1.3.1 Approximating Calculus with Algebra . . . . . . . . 26

1.3.2 Marching Schemes . . . . . . . . . . . . . . . 29

Problems . . . . . . . . . . . . . . . . 33

2 Basic Finite-Difference Methods 35

2.1 Accuracy and Consistency . . . . . . . . . . . . . 36

2.2 Stability and Convergence . . . . . . . . . . . . . 39

2.2.1 The Energy Method . . . . . . . . . . . . . . . 41

2.2.2 Von Neumann's Method . . . . . . . . . . . . . . . 43

2.2.3 The Courant-Fredrichs-Lewy Condition . . . . . . . . 45

2.3 Time-Differencing . . . . . . . . . . . . . . . 47

2.3.1 The Oscillation Equation: Phase-Speed and Amplitude Error . . . . . . . . . . . . . 48 
2.3.2 Single-Stage Two-Level Schemes _ . . . . . . . . . . 50

2.3.3 Multistage Methods . . . . . . . . . . . . . 53

2.3.4 Three-Level Schemes . . . . . . . . . . . . . . . 56

2.3.5 Controlling the Leapfrog Computational Mode . . . . . 60

2.3.6 Higher-Order Schemes . . . . . . . . . . . . . 65

2.4 Space-Differencing . . . . . . . . . . . . . . . . 72

2.4.1 Differential-Difference Equations and Wave Dispersion . . . . . . . . . . 73

2.4.2 Dissipation, Dispersion, and the Modified Equation . . . 80

2.4.3 Artificial Dissipation . . . . . . . . . . . . . . 82

2.4.4 Compact Differencing . . . . . . . . . . . . . . . 86

2.5 Combined Time- and Space-Differencing _ . . . . . . . . . . 89

2.5.1 The Discrete-Dispersion Relation . . . . . . . . . . . 91

2.5.2 The Modified Equation . . . . . . . . . . . . . . . . . 94

2.5.3 The Lax-Wendroff Method . . . . . . . . . . . . 95

2.6 Summary Discussion of Elementary Methods . . . . . . . . . . 99

Problems . . . . . . . . . . . . . . . . . . 101

3 Beyond the One-Way Wave Equation 107

3.1 Systems of Equations _ . . . . . . . . . . . . . . 107

3.1 .1 Stability . . . . . . . . . . . . . . . . 108

3.1.2 Staggered Meshes . . . . . . . . . . . . . . . . 113

3.2 Three or More Independent Variables . . . . . . . . . . . . 117

3.2.1 Scalar Advection in Two Dimensions . . . . . . . . . 117

3.2.2 Systems of Equations in Several Dimensions . . . . . . . 126

3.3 Splitting into Fractional Steps . . . . . . . . . . . . . . . . 129

3.3.1 Split Explicit Schemes . . . . . . . . . . . . . . . . 130

3.3.2 Split Implicit Schemes . . . . . . . . . . . . . . . . . 132

3.3.3 Stability of Split Schemes . . . . . . . . . . . . . 134

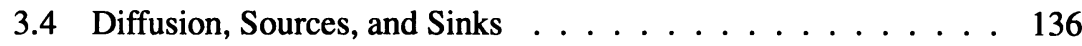

3.4 .1 Pure Diffusion . . . . . . . . . . . . . . . . 136

3.4.2 Advection and Diffusion . . . . . . . . . . . . . . . 138

3.4.3 Advection with Sources and Sinks . . . . . . . . . . . . 144

3.5 Linear Equations with Variable Coefficients . . . . . . . . . 147

3.5 .1 Aliasing Error . . . . . . . . . . . . . . . 148

3.5.2 Conservation and Stability . . . . . . . . . . . . . 154

3.6 Nonlinear Instability . . . . . . . . . . . . . . . . . . . . . . 159

3.6.1 Burgers's Equation . . . . . . . . . . . . . . . . . . 159

3.6.2 The Barotropic Vorticity Equation . . . . . . . . . . 163

Problems . . . . . . . . . . . . . . . . . . . . . . 167 
4 Series-Expansion Methods 173

4.1 Strategies for Minimizing the Residual . . . . . . . . . . . . 173

4.2 The Spectral Method . . . . . . . . . . . . . . . . . . 176

4.2.1 Comparison with Finite-Difference Methods . . . . . . . 177

4.2.2 Improving Efficiency Using the Transform Method . . . 184

4.2.3 Conservation and the Galerkin Approximation . . . . . . 189

4.3 The Pseudospectral Method . . . . . . . . . . . . . . 191

4.4 Spherical Harmonics . . . . . . . . . . . . . . . . . . . . . . 195

4.4.1 Truncating the Expansion _ . . . . . . . . . . . 197

4.4.2 Elimination of the Pole Problem . . . . . . . . . . . 200

4.4.3 Gaussian Quadrature and the Transform Method . . . . . 202

4.4.4 Nonlinear Shallow-Water Equations . . . . . . . . . . 207

4.5 The Finite-Element Method . . . . . . . . . . . . . . . . 212

4.5.1 Galerkin Approximation with Chapeau Functions . . . . 214

4.5.2 Petrov-Galerkin and Taylor-Galerkin Methods . . . . . 216

4.5.3 Quadratic Expansion Functions . . . . . . . . . . 219

4.5.4 Hermite-Cubic Expansion Functions . . . . . . . . . . 226

4.5.5 Two-Dimensional Expansion Functions . . . . . . . 231

Problems . . . . . . . . . . . . . . . . . . . . 234

5 Finite Volume Methods 241

5.1 Conservation Laws and Weak Solutions . . . . . . . . . . . 243

5.1.1 The Riemann Problem . . . . . . . . . . . . . . . . . 244

5.1.2 Entropy-Consistent Solutions . . . . . . . . . . . . 246

5.2 Finite-Volume Methods and Convergence . . . . . . . . . . . 249

5.2 .1 Monotone Schemes . . . . . . . . . . . . . . . 251

5.2 .2 TVD Methods . . . . . . . . . . . . . . . 252

5.3 Discontinuities in Geophysical Fluid Dynamics . . . . . . . . 254

5.4 Flux-Corrected Transport . . . . . . . . . . . . . . . . 257

5.4.1 Flux Correction: The Original Proposal . . . . . . . . 259

5.4.2 The Zalesak Corrector . . . . . . . . . . . . . . . . 260

5.4 .3 Iterative Flux Correction . . . . . . . . . . . . . 263

5.5 Flux-Limiter Methods . . . . . . . . . . . . . . . . 263

5.5.1 Ensuring That the Scheme Is TVD . . . . . . . . . . 264

5.5 .2 Possible Flux Limiters . . . . . . . . . . . . . . . . 267

5.5.3 Flow Velocities of Arbitrary Sign . . . . . . . . . . 271

5.6 Approximation with Local Polynomials . . . . . . . . . . . 272

5.6.1 Godunov's Method . . . . . . . . . . . . . . . . . 272

5.6.2 Piecewise-Linear Functions . . . . . . . . . . . . . . 274

5.7 Two Spatial Dimensions . . . . . . . . . . . . . . . . . 277

5.7.1 FCT in Two Dimensions . . . . . . . . . . . . . . . 277

5.7.2 Flux-Limiter Methods for Uniform 2-D Flow . . . . . . 279

5.7.3 Nonuniform Nondivergent Flow . . . . . . . . . . . 282

5.7 .4 A Numerical Example . . . . . . . . . . . . . . . . 284

5.7.5 When Is a Flux Limiter Necessary? . . . . . . . . . 291 
5.8 Schemes for Positive Definite Advection . . . . . . . . . . . . 292

5.8 .1 An FCT Approach . . . . . . . . . . . . . . . . 293

5.8.2 Antidiffusion via Upstream Differencing . . . . . . . . 294

5.9 Curvilinear Coordinates . . . . . . . . . . . . . . . 296

Problems . . . . . . . . . . . . . . . . 297

6 Semi-Lagrangian Methods 303

6.1 The Scalar Advection Equation . . . . . . . . . . . . 305

6.1.1 Constant Velocity .............. . 305

6.1 .2 Variable Velocity ................ 310

6.2 Forcing in the Lagrangian Frame . . . . . . . . . . . . . . . 313

6.3 Systems of Equations _. . . . . . . . . . . . . . . . 318

6.3.1 Comparison with the Method of Characteristics . . . . 318

6.3.2 Semi-implicit Semi-Lagrangian Schemes . . . . . . . 320

6.4 Alternative Trajectories . . . . . . . . . . . . . . . . 324

6.4.1 A Noninterpolating Leapfrog Scheme . . . . . . . . . 325

6.4.2 Interpolation via Parametrized Advection . . . . . . . 327

6.5 Eulerian or Semi-Lagrangian? . . . . . . . . . . . . . 330

Problems ..................... 331

7 Physically Insignificant Fast Waves 335

7.1 The Projection Method . . . . . . . . . . . . . . . . 336

7.1.1 Forward-in-Time Implementation . . . . . . . . . 337

7.1.2 Leapfrog Implementation . . . . . . . . . . . . 339

7.1.3 Solving the Poisson Equation for Pressure . . . . . . . . 340

7.2 The Semi-implicit Method . . . . . . . . . . . . . . . 342

7.2.1 Large Time Steps and Poor Accuracy . . . . . . . . 343

7.2.2 A Prototype Problem . . . . . . . . . . . . . 345

7.2.3 Semi-implicit Solution of the Shallow-Water Equations . 347

7.2.4 Semi-implicit Solution of the Euler Equations . . . . . . 350

7.2.5 Numerical Implementation . . . . . . . . . . . . . 356

7.3 Fractional-Step Methods . . . . . . . . . . . . . . 359

7.3.1 Complete Operator Splitting . . . . . . . . . . . . 359

7.3.2 Partially Split Operators . . . . . . . . . . . 365

7.4 Summary of Schemes for Nonhydrostatic Models . . . . . . . . 371

7.5 The Hydrostatic Approximation . . . . . . . . . . . . . 372

7.6 Primitive Equation Models . . . . . . . . . . . . . . . 374

7.6.1 Pressure and $\sigma$ Coordinates . . . . . . . . . . . 375

7.6.2 Spectral Representation of the Horizontal Structure . . . 379

7.6.3 Vertical Differencing . . . . . . . . . . . 381

7.6.4 Energy Conservation . . . . . . . . . . . . 383

7.6.5 Semi-implicit Time-Differencing . . . . . . . . 387

Problems . . . . . . . . . . . . . . . . . . . . 389 
8 Nonreflecting Boundary Conditions 395

8.1 One-Dimensional Flow . . . . . . . . . . . . . . . . . . . . . 397

8.1.1 Well-Posed Initial-Boundary Value Problems. . . . . . . 397

8.1.2 The Radiation Condition . . . . . . . . . . . . . . . . 400

8.1.3 Time-Dependent Boundary Data . . . . . . . . . . . . 401

8.1.4 Reflections at an Artificial Boundary-

The Continuous Case . . . . . . . . . . . . . . . . 402

8.1.5 Reflections at an Artificial Boundary-

The Discretized Case . . . . . . . . . . . . . . . 403

8.1.6 Stability in the Presence of Boundaries . . . . . . . . . 409

8.2 Two-Dimensional Shallow-Water Flow . . . . . . . . . . . 412

8.2.1 One-Way Wave Equations . . . . . . . . . . . . . . . 414

8.2.2 Numerical Implementation . . . . . . . . . . . . . . 419

8.3 Two-Dimensional Stratified Flow . . . . . . . . . . . . . . . . 419

8.3.1 Lateral Boundary Conditions . . . . . . . . . . . . . . 420

8.3.2 Upper Boundary Conditions . . . . . . . . . . . . . . . 424

8.3.3 Numerical Implementation of the Radiation Upper

Boundary Condition . . . . . . . . . . . . . . . 429

8.4 Wave-Absorbing Layers . . . . . . . . . . . . . . . . . 431

8.5 Summary . . . . . . . . . . . . . . . . 436

Problems . . . . . . . . . . . . . . . . . 437

Appendix Numerical Miscellany 439

A.1 Finite-Difference Operator Notation . . . . . . . . . . . . . 439

A.2 Tridiagonal Solvers . . . . . . . . . . . . . . . . . 440

A.2.1 Code for a Tridiagonal Solver . . . . . . . . . . . . . 440

A.2.2 Code for a Periodic Tridiagonal Solver . . . . . . . . 441

Bibliography 443

Index 457 